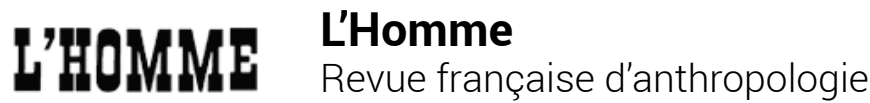

166 | avril-juin 2003

Malinowski, Faulkner. Culture et cognition. Souvenir et héritage

\section{Et si nous prenions nos désirs en compte?}

André Micoud, Laurence Bérard, Philippe Marchenay et Michel Rautenberg

\section{(2) OpenEdition}

1 Journals

Édition électronique

URL : http://journals.openedition.org//homme/228

DOI : $10.4000 /$ /homme.228

ISSN : 1953-8103

Éditeur

Éditions de l'EHESS

\section{Édition imprimée}

Date de publication : 30 juin 2003

Pagination : 235-238

ISBN : 2-7132-1805-5

ISSN : 0439-4216

Référence électronique

André Micoud, Laurence Bérard, Philippe Marchenay et Michel Rautenberg, «Et si nous prenions nos désirs en compte ? », L'Homme [En ligne], 166 | avril-juin 2003, mis en ligne le 08 septembre 2008, consulté le 19 avril 2019. URL : http://journals.openedition.org//homme/228 ; DOI : 10.4000/ Ihomme.228 


\title{
Et si nous prenions nos désirs en compte?
}

\author{
André Micoud, Laurence Bérard, \\ Philippe Marchenay \& Michel Rautenberg
}

1

est gratifiant pour les auteurs d'un ouvrage de pouvoir lire des analyses de leur production; surtout quand celles-ci sont faites par des lecteurs perspicaces. On remerciera donc en premier lieu les deux auteurs de cet «À propos » de s'être livrés à ce travail de décryptage qui, à tout le moins, relève d'une certaine acribie. Les questions qu'ils nous posent sont souvent de vraies et bonnes questions. Et pour autant, il nous semble finalement - et précisément par la dernière note infra-paginale que leur lecture, sans doute trop orientée par un a priori (à moins que ce soit par une fin pratique), pêche à vouloir trop prouver. Certes, ils ont bien lu le livre et tous les articles qu'il contient, mais peut-être n'ontils pas voulu être assez attentifs à l'avantpropos qui, signé par Denis Chevallier, vient en grande partie ruiner leur thèse répétée à l'envi : à savoir que de n'avoir pas su objectiver notre propre position, nous serions devenus des ethnologues à la solde de leurs commanditaires. Qu'on relise donc d'abord cet avant-propos (et aussi tout le paratexte) et l'on y verra que tout est dit sur ces fameuses conditions de production que l'on nous reproche d'avoir voulu cacher. Combien de travaux de ce genre prennentils la peine d'être aussi diserts sur ce point?
Il reste que, nonobstant cette partialité, il convient de lire cette critique avec toute l'attention qu'elle mérite. C'est en tout cas ce à quoi nous voulons nous employer dans cette réponse. Mais tout d'abord une précision liminaire s'impose. Certes, la question qui nous est posée - à nous éditeurs scientifiques de cet ouvrage - est, à l'évidence, tout à fait recevable. Elle revient à nous demander: d'où parlez-vous? Sauf que cette évidence justement, peut-être d'avoir trop servi, ne peut plus empêcher la répartie de venir aussitôt: et vous-mêmes? On sait trop cependant que s'engager dans cette voie ne pourrait que rouvrir la sempiternelle querelle opposant des recherches désintéressées et lucides sur leurs conditions d'effectuation à celles par trop dépendantes de financeurs qui, plus ou moins subrepticement, en viennent à dicter leur loi et à occulter leur pouvoir. Nous pensons que de ce débat, aussi vieux que celui sur la légitimité, il n'y a qu'une seule manière de sortir: celle qui consiste à ce que chacun des protagonistes expose à quelle loi, pour être libre, il choisit de s'assujettir. En répondant à cette question, chacun témoignera alors ipso facto de son attachement à telle ou telle communauté ou, à tout le moins, explicitera comment il hiérarchise ses appar- 
tenances aux différents mondes auxquels, et quoi qu'il en veuille, il se trouve appartenir de fait. Tel sera bien le sens de notre explication, in fine, de l'emploi par nous de ce pronom personnel «nos », dont le caractère ambigu n'a pas échappé à nos deux lecteurs puisque c'est autour de lui qu'ils ont forgé leur titre en forme de flèche.

Le texte proprement dit, en effet, n'est que le déploiement de ce qui est contenu de façon ramassée dans l'intitulé de cet « $\AA$ propos ». Certes, le jugement inclus dans ce trait liminaire est d'abord tempéré, qui accorde à certains d'entre nous d'avoir, par ailleurs, fait allégeance «à juste titre » à la bonne «vision du constructivisme de la tradition »; qui leur accorde aussi d'avoir su témoigner dans d'autres publications qu'ils savaient qu'une recherche digne de ce nom devait toujours inclure l'analyse de ses propres conditions de possibilité. Dont acte. On saura gré à nos juges d'avoir eu de bonnes lectures. Sauf que là nous aurions fauté. En effet, partis d'une bonne « analyse constructiviste du patrimoine rural », nous nous serions ensuite mis ici au service d'une « production [en bonne et due forme] du patrimoine rural ». La critique est sévère qui tombe sous le coup de l'accusation la plus infamante pour les gens de la discipline où nous sommes rangés: celle de naturaliser une catégorie.

Pourquoi alors une telle dérive de la part de chercheurs d'habitude plus circonspects? La réponse est suggérée sous la forme d'une autre critique, plus «stratégique » celle-là, et menée à partir de l'analyse de la diffusion de nos propos. Selon celle-ci en effet, tandis que notre ouvrage Campagne de tous nos désirs (« financé par la Mission du patrimoine ethnologique du ministère de la Culture », est-il subtilement rappelé) est réservé à nos pairs, la livraison du numéro d'Autrement (qui « réunit peu ou prou le même groupe de chercheurs ») destinée «à un public plus large », « sonne [quant à elle] comme un manifeste en faveur du patrimoine rural $\gg$. Ainsi donc la critique ne vise-t-elle plus que les seuls auteurs du livre en question mais un «groupe de chercheurs ». Ceux-ci, c'est-à-dire les mêmes qui sont « peu ou prou » présents ici et là, et dont les deux publications sont toujours financées, ici par la Mission du patrimoine ethnologique, et là par l'opération « Patrimoine rural » du ministère de l'Agriculture et de la Pêche, joueraient donc sur les deux tableaux. D'un côté, grâce à la logique constructiviste, ils participeraient à la légitimation d'une démarche dans le champ scientifique, quand de l'autre, celle de l'écriture naturaliste, ils viendraient assurer le bien-fondé de ces politiques de patrimonialisation « essentialisées par l'ethnologie et authentifiées par l'histoire ». Nous ne sommes donc plus seulement quelques ethnologues égarés, mais un groupe de chercheurs. Davantage même, un groupe de chercheurs qui, en usant de « deux registres discursifs » pour s'adresser à « deux publics différents », n'est plus très loin de ressembler à une clique quand il irait même jusqu'à lancer des appels aux chercheurs « à investir les instances institutionnelles ». Loin donc que d'être des ethnologues naiffs (encore un peu de logique constructiviste s'il vous plait), nous serions bien plutôt ceux qui accepteraient de travailler à ce que les désirs de la puissance publique se transforment en réalité. Ce que les auteurs de cet «À propos» nous reprochent peut donc être résumé ainsi : à défaut de nous être posés la question de savoir à quelle entreprise nous participions (à l'insu de notre plein gré ou en connaissance de cause), alors il ne pouvait pas nous apparaître à quel point ce prurit patrimonialisant n'était que l'effet des politiques concertées des différents ministères (de la Culture, de l'Agriculture, du Tourisme, de la DATAR...). Pour nos accusateurs donc, ce défaut d'auto-objectivation n'est plus seulement imputable à une insuffisance professionnelle, il est structurel à la situation.

La voie étant ainsi ouverte, la condamnation peut alors se déployer. Elle le fait sur deux fronts. Le premier, déjà amplement labouré, de la non-explicitation par les chercheurs de la place qu'ils occupent dans la patrimonialisation du rural et qui «s'avère dommageable ». Et le second qui, en disant comment désigner cette place - « Le maillon manquant qui unirait tous ces terrains est la scène nationale »-, nous accuse précisément d'avoir accepté de composer avec l'ambiguïté 
inhérente à la Mission du patrimoine ethnologique, supposée être le grand ordonnateur de toute cette entreprise. Notre faute ne serait pas tant celle d'être des ethnologues ayant pris leur désir pour la réalité (encore que nous puissions en même temps être crédités de ce pouvoir proprement démiurgique de « créer ex nihilo des appropriations collectives des espaces et des produits ruraux »), mais celle de nous être laissés manipuler, permettant ainsi aux institutions nationales de réaliser les leurs.

On aura reconnu sans peine quelle est cette position qui revient à dire que toute la patrimonialisation relève en fin de compte des politiques publiques qui l'instrumentalisent : constitutif de la sociologie critique, elle est celle consistant à soutenir qu'il n'est pas possible qu'il y ait des gens capables de se conduire spontanément en réponse à une situation. Toujours et en tous lieux, des appareils les dominent symboliquement pour dicter leur réponse. Et l'on aura reconnu aussi quel est le désir qui soutient et qui anime cette prise de position: celui, éminemment paradoxal et pourtant toujours recommencé, qui lui commande de croire à ce qu'il sait être impossible. Or, pour nous, le geste de séparation par lequel cette sociologie s'instaure - entre le spontané d'un côté, toujours illusoire et toujours espéré, et la domination de l'autre, toujours là et toujours à dénoncer - est en même temps celui qui l'exile, qui condamne ses servants à l'impossible neutre (du latin ne uter, ni... ni) et qui, à défaut de pouvoir les installer soit dans la stature du maitre (cf. note 6) soit dans celle de porte-parole des humiliés (« les inactifs qui subissent l'action »), ne peut que les faire osciller sans cesse entre l'une et l'autre identifications.

Mais pourquoi répondre ici de cette manière? Parce que, dans ce texte précisément, les indices sont nombreux qui permettent de déceler la force de cet impensé constitutif, de ce véritable point aveugle (et du désir qui y est attaché). On en a décelé au moins quatre occurrences. Reproche nous ait fait d'abord, « Là où le bât blesse », de « donner à croire à une capacité d'auto-patrimonialisation des lieux, ou encore à une auto- nomie de la scène locale vis-à-vis de la scène nationale », quand il est dit plus loin que « ce que ces travaux décrivent comme la nouveauté récente $[. .$.$] n'a pour nous pas grand-$ chose de spontané, n'étant qu'un effet de la politique d'aménagement du territoire ». Reproche ensuite, sous la forme d'une fausse question ("La causalité remonte-t-elle du terrain vers la recherche ou descend-elle du chercheur vers le terrain via les ministères ? »), de nous être faits les relais de la domination. Reproche encore qui mobilise toujours la même opposition (« labellisation versus endogène ») quand il est dit plus loin que « l'invention récente d'une patrimonialisation des produits de terroir [...] nous semble plus la conséquence d'une re-labellisation administrative et scientifique [...] que le signe d'un changement endogène, local, des rapports aux objets ruraux $»$.

On le voit, un même système d'oppositions est utilisé de façon récurrente qui, posant l'existence antérieure d'une « politique patrimoniale - étatique ou communale - construite par les chercheurs », permet de nous accuser ensuite de ne pas l'avoir reconnue « comme un fait structurant les données recueillies ». Tout le raisonnement qui suit à partir de ce qui est à proprement parler une mise en scène, et une mise en histoire «nationale », n'est en fait que la reprise de cette antienne. Comparés aux érudits et sociétés savantes du XIX siècle, nous voilà ramenés au statut de « principaux diffuseurs de modèles culturels de promotions des territoires qui initient largement ce mouvement de patrimonialisation ». D'ailleurs, de n'avoir pas consacré de travaux aux mouvements régionalistes dans l'entre-deux-guerres suffit à nous faire passer pour des gens qui, implicitement, ne considéreraient « qu'il n'y aurait patrimonialisation que quand l'État décide qu'il va y avoir du patrimoine ». Mais c'est encore trop peu quant à ignorer la multiplicité des inventions des politiques culturelles à partir des années 1980 (d'après Urfalino) et, du coup, à reprendre « sans réflexivité » cette «notion administrative de patrimoine», ce ne pouvait que nous condamner à être les commis de cette «compréhension républicaine et démocratique des faits culturels ». 
Sauf que ce n'est pas en ces termes (ainsi opposés) que nous pensons, et c'est de là que découle notre choix de ne pas nous distinguer de cela avec quoi nous avons partie liée. Pour autant, ni nous ne nous avançons sous une « identité masquée », ni ne nous identifions, au choix, soit « aux urbains dans leur ensemble », soit aux « ethnologues de la Mission du patrimoine ou aux fonctionnaires de la culture ». À l'écart de cette façon de faire de la recherche - celle que les deux auteurs appellent de leurs vœux et qui consisterait (gage d'une grande réflexivité?) à faire «une sociologie de l'ethnologie contemporaine » (et pourquoi pas une ethnologie de la sociologie de l'ethnologie etc. et ad nauseam?) - ce travail se range délibérément sous le registre du témoignage. Travail qui ne considère pas ses attaches comme des handicaps, mais comme attestation de son irréductible historicité.

Plutôt que d'ambitionner atteindre ce lieu où, parce qu'elles seraient objectivées, « ces stratégies inconscientes de leur savoir procurent [...] le moyen de tout expliquer et d'être conscient de tout $\rangle^{\prime}$, nous pensons plus simplement qu'il était de notre responsabilité de rendre compte. De rendre compte de quoi? De ce qu'un mouvement existe, qui emporte avec lui aussi bien les administrations que les chercheurs, aussi bien les opérateurs culturels que les animateurs d'associations locales de sauvegarde du patrimoine, aussi bien les agents des Parcs naturels régionaux que ceux qui cherchent un avenir pour des régions à l'écart, et qui veut redire «notre » campagne en d'autres termes. Que d'autres « acteurs »ceux qui parlent en termes « d'espace rural » notamment - aient du mal à s'y reconnaître n'enlève rien à l'affaire. Les reconfigurations sociales et symboliques, jamais, ne sont unanimes. Nulle part nous avons écrit que ce mouvement avait déjà réussi, ni non plus que nous savions où il allait. Et, accepterait-on cette dichotomie entre ceux d'en haut et ceux d'en bas, nulle part non plus nous avons décrété savoir où il avait bien pu commencer. Que des ethnologues d'État soient là pour participer à la construction du patrimoine rural qui sied aux appareils du même nom, eh bien soit. Sauf qu'ils ne sont ni les seuls ni les premiers, loin de là! II y a bien longtemps que nous le savons: les gens des sciences humaines - et rappelons que dans notre cas, ce ne sont pas seulement des ethnologues qui sont embarqués, mais aussi des géographes, des historiens, des sociologues... - ne font jamais qu'arriver après les « acteurs »; ici après ceux qui, en peine de construire un avenir pour des espaces en déshérence, ont commencé à imaginer les mille et une façons d'y parvenir.

Ramenés de la sorte à cette simple place de témoins, tout au plus pouvons-nous dire que ces ainsi nommés « nos désirs 》, multiformes et inassignables, ont déjà transformé « l'espace rural », comme le désignent nos auteurs, en le faisant re-connaitre à nouveau sous le vieux mot de « campagne ». Les monographies présentées dans ce livre ne font donc, chacune à leur façon, que prendre en compte ce mouvement qui, volens nolens, « nous » emporte tous pour - à propos des campagnes européennes en général et de la campagne française en particulier non pas refaire la république, mais, peutêtre, la réinterpréter pour l'énoncer à nouveau, en français cette fois, comme une nouvelle « chose commune ».

I. Michel de Certeau, L'Invention du quotidien. I. L'art de faire, Paris, UGE, |980: |0| («|0/|8»). 